\title{
Objective analysis of cancer stem cell marker expression using immunohistochemistry
}

\author{
T. J. Miller ${ }^{1,2}$, M. J. McCoy ${ }^{2,3}$, C. Hemmings ${ }^{1,4}$, M. K. Bulsara ${ }^{5}$, \\ B. Iacopetta ${ }^{1}$ and C. F. Platell ${ }^{1,2}$ \\ ${ }^{1}$ School of Surgery, University of Western Australia, Nedlands, ${ }^{2}$ Colorectal Research Unit, St \\ John of God Subiaco Hospital, Subiaco, ${ }^{3}$ School of Medicine and Pharmacology, University \\ of Western Australia, Nedlands, ${ }^{4}$ St John of God Pathology, Subiaco, and ${ }^{5}$ Institute for Health \\ Research, University of Notre Dame, Fremantle, Australia
}

\begin{abstract}
Summary
Analysis of immunohistochemical expression is often a subjective and semiquantitative process that can lead to the inconsistent reporting of results. To assess the effect that region selection and quantification method have on results, five different cancer stem cell markers were used in this study to compare tissue scoring with digital analysis methods that used three different tissue annotation methods. Samples of tumour and normal mucosa were used from 10 consecutive stage II colon cancer patients and stained for the putative cancer stem cell markers ALDH1, CD44v6, CD133, Lgr5 and SOX2. Tissue scoring was found to have considerably different results to digital analysis with the three different digital methods harbouring concordant results overall. However, SOX2 on normal tissue and CD133 on tumour and normal tissue produced discordant results which could be attributed to the different regions of tissue that were analysed. It is important that quantification method and selection of analysis areas are considered as part of study design to ensure that reproducible and consistent results are reported in the literature.
\end{abstract}

Key words: Colorectal cancer; cancer stem cell; immunohistochemistry; ALDH1; CD44v6; CD133; Lgr5; SOX2.

Received 7 April, revised 8 August, accepted 14 September 2016 Available online 29 November 2016

\section{INTRODUCTION}

The existence of cancer stem cells (CSC) in colorectal cancer (CRC) is currently an active area of investigation. Following the initial descriptions of $\mathrm{CSC}^{1,2}$ several studies have investigated their role in human $\mathrm{CRC}$ by using immunohistochemical (IHC) analysis to quantify the expression of CSC biomarkers in tumour samples.

The method of analysis varies between studies but semiquantitative scoring of biomarker expression is the most widely used. However, this is somewhat subjective and can result in inter-observer variability. ${ }^{3,4}$ Different interpretations of staining intensity, or variations in scoring methods, may also contribute to contrasting findings being reported in the literature. ${ }^{5-9}$ For example, whereas one study counting the number of glands expressing the glycosylated transmembrane protein CD133 found that high expression was independently prognostic for poor cancer-specific survival, another study using a scale of $0-3$ for positive tumour staining found that CD133 was not a significant prognostic factor in CRC. ${ }^{7}$ These studies analysed different areas of the tumour samples and used different cut-off scores, possibly impacting the final result. ${ }^{6,7}$ Studies using tissue microarrays (TMAs) have been reported to result in more false-negative associations between CD133 expression and CRC patient survival, ${ }^{10}$ implying that tissue selection is an important factor. More objective and defined methods for analysis and tissue selection are likely to improve the reproducibility of published results.

Automated digital platforms now offer the possibility for objective analysis of protein expression in tissue samples. Algorithms supplied with the software packages allow quantification of the percentage of positivity by tissue area, or by cell count. In this study we aimed to compare different tissue annotation methods and to assess the expression of five CSC biomarkers using the Aperio Imagescope software platform.

\section{MATERIALS AND METHODS}

Patients and samples

Formalin fixed, paraffin embedded samples of tumour and adjacent normal mucosa were retrieved from 10 consecutive patients who underwent potentially curative resection in 2014 for stage II colon cancer at the St John of God Hospital, Subiaco, Western Australia. The study was approved by the St John of God Healthcare and University of Western Australia Human Research Ethics Committees. All patients used in this study consented to the use of their biological samples.

\section{Immunohistochemistry}

Six serial sections were cut from each block and stained with haematoxylin and eosin (H\&E) and for five different CSC markers associated with colorectal cancer: CD133, aldehyde dehydrogenase-1 (ALDH1), CD44-variant -6 (CD44v6), leucine-rich repeating G-protein coupled receptor-5 (Lgr5) and sex-determing region-Y homeobox-2 (SOX2). Antigen retrieval was performed in $10 \mathrm{mM}$ citrate buffer ( $\mathrm{pH} \mathrm{6.0)}$ for $6 \mathrm{~min}$ at $121^{\circ} \mathrm{C}$ using the DakoCytomation Pressure Cooker (Dako, Denmark). Incubation with primary antibody was performed at room temperature for $30 \mathrm{~min}$ for CD44v6 (clone VFF-18; 1:1000 dilution; Abcam, Australia), CD133 (clone AC133; 1:50 dilution; Miltenyi Biotec, Australia) and Lgr5 (clone MC-1235; 1:200 dilution; MBL International, USA) and for 1 hour for ALDH1 (clone 44; 1:1000 dilution; BD Biosciences, USA) and SOX2 (clone EPR3131; 1:50 
dilution; Abcam). Endogenous peroxide was blocked using Peroxidazed 1 (Biocare Medical, USA) and non-specific IgG interactions were blocked using Background Sniper (Biocare Medical). Immunostaining was then completed using the EnVision-HRP/DAB System (Dako). SOX2 antibody required use of the EnVision Rabbit Link reagent (Dako) for amplification. All sections were lightly counterstained in Mayers haematoxylin (Hurst Scientific, Australia) before dehydration and mounting. Slides were scanned using a high resolution digital scanner (Aperio Scanscope XT; Leica Biosystems, Australia).

\section{Quantification of marker expression}

Three regions of interest (ROI) for each of tumour and normal epithelium were selected at medium power $(20 x)$ for analysis. To avoid being influenced by the CSC marker staining pattern, regions for analysis were first selected using $H \& E$ images and these areas were then selected on the corresponding IHC-stained images (Fig. 1). For each stain, five different quantification methods were performed.

1. Scoring: The three ROIs were scored semi-quantitatively for staining intensity and proportions of cells stained on a scale of $0-3$ by one observer (TM). Separate scores were given for epithelium and stroma.

2. Grid-plot: A $2 \times 2 \mathrm{~mm}$ grid was overlayed on each ROI and each intersecting point was scored using the same scoring system, noting the tissue type (epithelium/stroma/lamina propria).

3. Digital ROI: The Aperio colour deconvolution algorithm v9 (Leica Biosystems) was used to objectively quantify the percentage area of positive staining for CD133, CD44v6, ALDH1 and Lgr5 on each ROI. The nuclear quantification algorithm v9 was used to calculate the percentage of cells positive for SOX2. Luminal areas that contained cell debris were excluded from this analysis.

4. Digital full-face: Tumour or normal area of tissue was traced at fullface with the same algorithms used to quantify expression.
5. Digital whole section: Algorithms were used on the whole of each section without any tracing or annotation.

\section{Calculation of $\mathbf{H}$-scores}

To compare the manual ROI scoring method to the three digital analysis methods, H-scores were calculated to give a score from 0 to 300 based on intensity and proportions of expression for each marker and method. For the manual 'Scoring' method, and for SOX2 expression quantified using any of the digital analysis methods, $\mathrm{H}$-scores were calculated using the following formula:

$\mathrm{H}$-score $=(3 \times$ percentage of $3+$ cells $)+(2 \times$ percentage of $2+$ cells $)+(1 \times$ percentage of +1 cells).

For ALDH1, CD44v6, CD133 and Lgr5 analysed using the digital analysis methods, H-scores were calculated using the following formula:

$\mathrm{H}$-score $=(3 \times$ percentage area of strong staining $)+(2 \times$ percentage area of moderate staining $)+(1 \times$ percentage area of weak staining $)$.

\section{Statistical analysis}

Kendall's W statistic and Kendall's tau-b concordance coefficients were calculated to determine the level of agreement between the analysis methods used in this study. Overall $p$ values of $<0.05$ were considered significant where the Bonferroni adjustment $(p<0.017)$ was made for pairwise comparisons. Bland-Altman plots were constructed for pairwise comparisons as previously described. ${ }^{11}$ SAS v9.4, STATA v14 and Graphpad Prism v6 were used for statistical analysis.

\section{RESULTS}

Representative IHC staining patterns for all markers are presented in Fig. 2.

The results of manually scoring ROIs for expression of each marker are reported in Supplementary Table 1
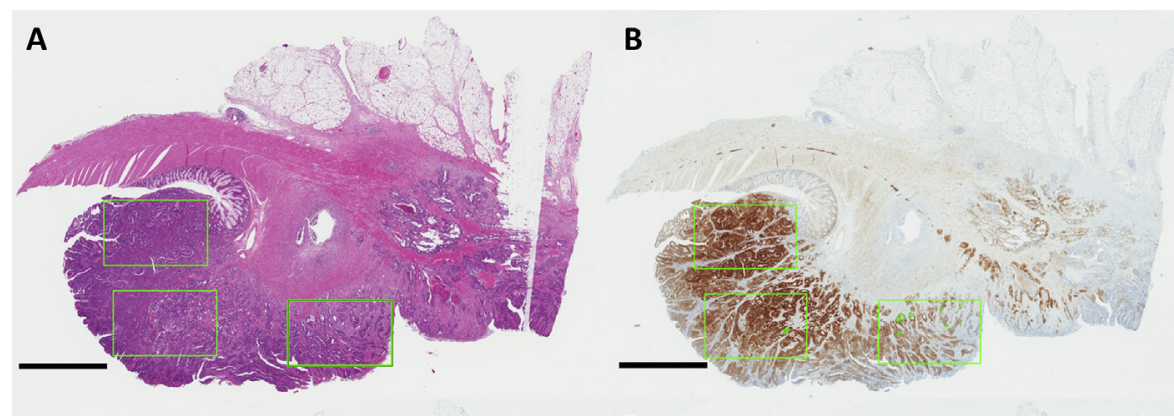

C

D

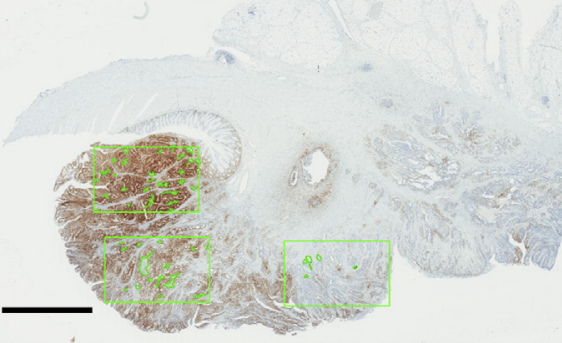

E

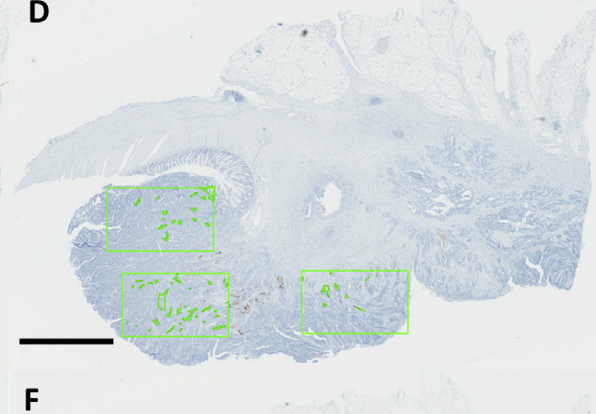

F

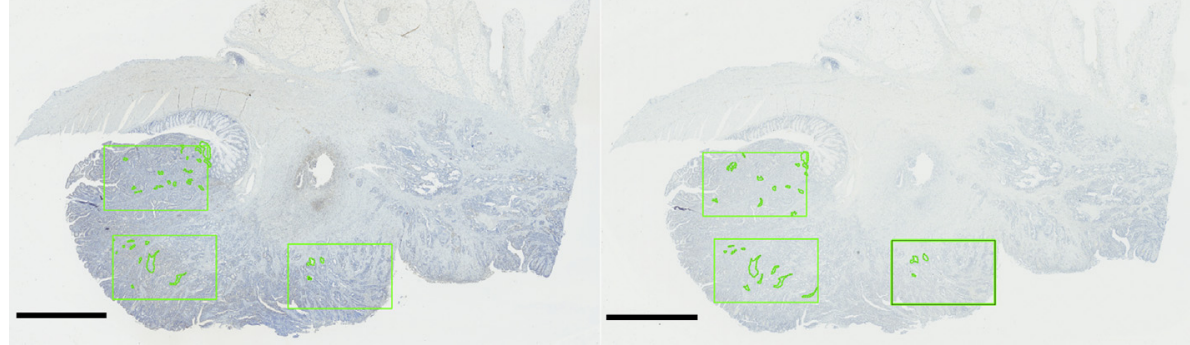

Fig. 1 Region of interest (ROI) selection for manual scoring and digital ROI methods. (A) Three regions of tumour or normal tissue were selected on the H\&E section and copied to all IHC images $(\mathrm{B}-\mathrm{F})$. Bar $=5 \mathrm{~mm}$. 


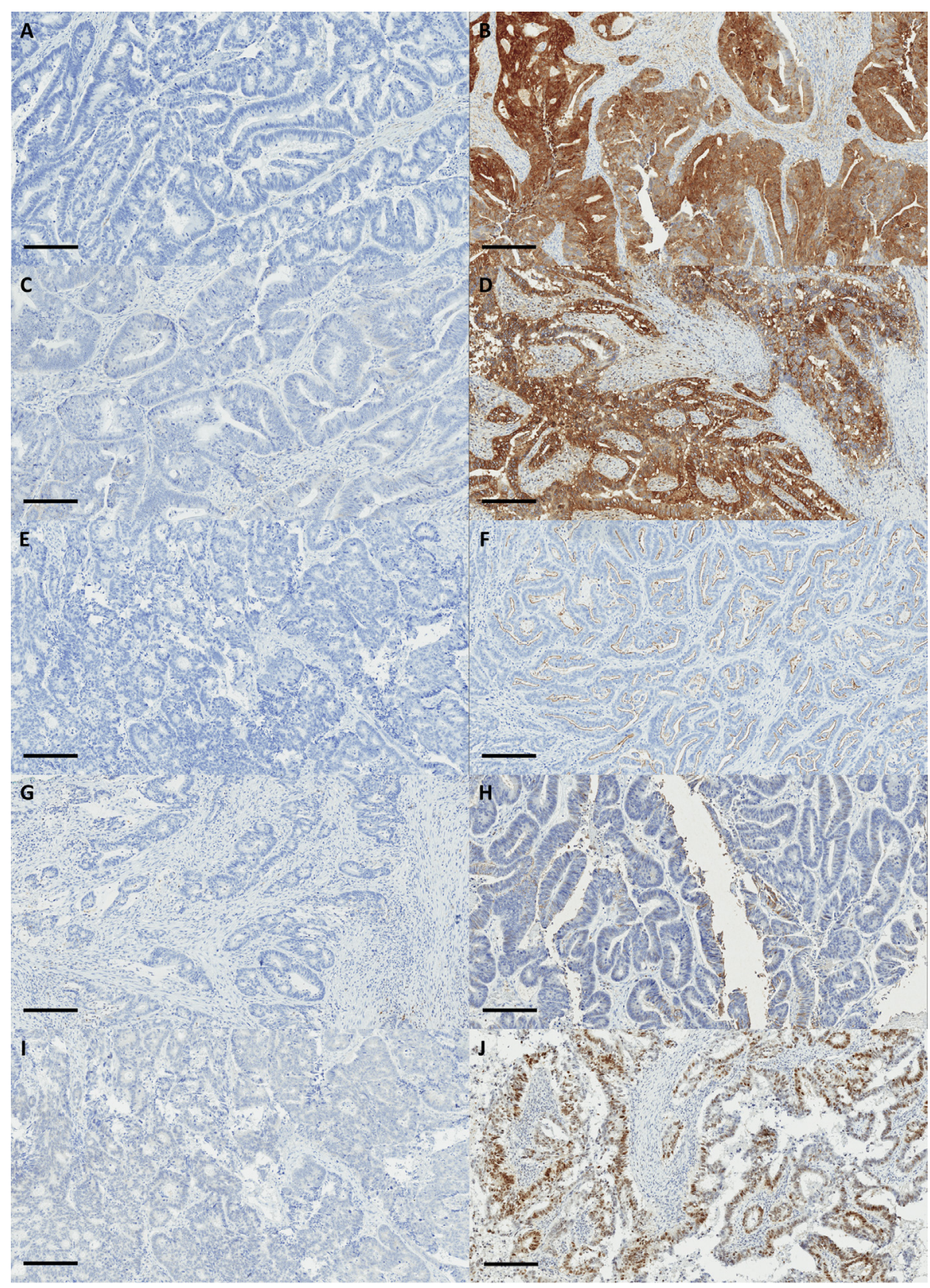

Fig. 2 Negative and positive staining for each immunostain in tumour samples. Representative areas of negative (A,C,E,G,I) and positive (B,D,F,H,J) staining for ALDH1 (A,B), CD44v6 (C,D), CD133 (E,F), Lgr5 (G,H) and SOX2 (I,J) in tumour samples. Bar = $200 \mu \mathrm{m}$.

(Appendix A) with $\mathrm{H}$-scores reported in Supplementary Table 2 (Appendix A). To compare the results of manual scoring against the digital analysis methods, cases were ranked from lowest to highest according to each analysis method using H-scores. Rankings based on CD133 expression in tumour tissue revealed considerable variation between the analysis methods (Table 1).

Following analysis of the first two cases, the grid-plot analysis method was judged not to be feasible due to the large amount of time required.

The percentage positive area was compared across the three digital analysis methods using Kendall's W concordance coefficient testing. The three methods produced mostly concordant results, however CD133 in both tumour $(\mathrm{W}=0.577, p=0.076)$ and normal tissue $(\mathrm{W}=0.558$, $p=0.090)$, as well as SOX2 in normal tissue $(\mathrm{W}=0.539$,
Table 1 Ranking of cases according to $\mathrm{H}$-scores for $\mathrm{CD} 133$ expression on tumour tissue assessed using each analysis method

\begin{tabular}{lcccc}
\hline Case & Full-face & Whole Section & ROI & Manual \\
\hline 5 & $\mathbf{1}$ & $\mathbf{1}$ & 2 & $\mathbf{1}$ \\
6 & 2 & 7 & $\mathbf{1}$ & $\mathbf{1}$ \\
9 & 3 & 4 & 7 & 8 \\
7 & 4 & 2 & $\mathbf{5}$ & $\mathbf{5}$ \\
10 & 5 & 5 & 8 & 7 \\
1 & 6 & $\mathbf{6}$ & $\mathbf{9}$ & $\mathbf{9}$ \\
8 & 7 & 9 & $\mathbf{3}$ & $\mathbf{6}$ \\
3 & 8 & 8 & $\mathbf{4}$ & $\mathbf{4}$ \\
4 & 9 & $\mathbf{1 0}$ & $\mathbf{1 0}$ & $\mathbf{1 0}$ \\
2 & $\mathbf{1 0}$ & & & \\
\hline
\end{tabular}

Table arranged by the ranks of the full-face digital analysis method. Equal ranks highlighted in bold.

ROI, region of interest. 
Table 2 Overall Kendall's W concordance coefficient test results comparing the three digital analysis methods

\begin{tabular}{llllll}
\hline \multirow{2}{*}{ Marker } & \multicolumn{2}{c}{ Tumour tissue } & & \multicolumn{2}{c}{ Normal tissue } \\
\cline { 2 - 3 } \cline { 5 - 6 } & Kendall's W & $p$ value & & Kendall's W & $p$ value \\
& & & & & \\
\hline ALDH1 & 0.9 & 0.004 & & 0.73 & 0.02 \\
CD44V6 & 0.96 & 0.002 & & 0.86 & 0.006 \\
CD133 & $\mathbf{0 . 5 8}$ & $\mathbf{0 . 0 7 6}$ & & $\mathbf{0 . 5 6}$ & $\mathbf{0 . 0 9}$ \\
Lgr5 & 0.88 & 0.005 & & 0.88 & 0.005 \\
Sox2 & 0.83 & 0.008 & & $\mathbf{0 . 5 4}$ & $\mathbf{0 . 1 0 4}$ \\
\hline
\end{tabular}

Non-significant $p$ values indicate discordant results (bold).

$p=0.104$ ) gave discordant results (Table 2). Discordant results were further investigated using Kendall's tau-b to perform pairwise comparisons for each method, as well as Bland-Altman plots (Supplementary Fig. 1, Appendix A). Although Bland-Altman plots suggested that all pairwise comparisons demonstrated agreement (Supplementary Fig. 1, Appendix A), Kendall's tau-b for the full-face versus whole section methods for CD133 expression in tumour tissue ( $\tau=0.6444, p=0.0095)$ was the only significantly concordant pair of the variables discordant in overall Kendall's W analysis (Table 3). The exclusion of luminal areas in the digital ROI method may explain the lower expression values for CD133 compared to other digital methods (Fig. 3). For normal tissue, the discordance is likely due to inclusion of more non-specific background staining in the whole section method (Fig. 4). The median percentage expression for discordant values in the overall analysis are presented for each method in Table 4.

When the Kendall's W concordance coefficient testing was performed to compare all four analysis methods using $\mathrm{H}$ scores, CD133 in tumour $(\mathrm{W}=0.612, p=0.009)$ and SOX2 in normal tissue $(\mathrm{W}=0.541, p=0.022)$ demonstrated improved concordance (Table 5). However, the improvement observed for CD133 in tumour tissue overall may be due to the manual ROI scoring method showing a high level of agreement with the digital ROI method $(\tau=0.854$, $p=0.0006)$. A large degree of discordance was observed for the pairwise comparisons of the manual ROI scoring method against the other digital analysis methods (Supplementary Table 3, Appendix A).

\section{DISCUSSION}

Tumour and normal mucosa samples from 10 consecutive stage II CRC patients were stained for five CSC markers that

Table 3 Pairwise Kendall's tau-b test results for samples that were discordant in overall Kendall's W test for the three digital analysis methods

\begin{tabular}{|c|c|c|c|c|c|c|}
\hline \multirow[t]{2}{*}{ Marker/Tissue } & \multicolumn{2}{|c|}{$\begin{array}{l}\text { ROI vs } \\
\text { full-face }\end{array}$} & \multicolumn{2}{|c|}{$\begin{array}{c}\text { ROI vs } \\
\text { whole section }\end{array}$} & \multicolumn{2}{|c|}{$\begin{array}{l}\text { Full-face vs } \\
\text { whole section }\end{array}$} \\
\hline & Tau-b & $p$ value & Tau-b & $p$ value & Tau-b & $p$ value \\
\hline CD133 Tumour & 0.33 & 0.18 & 0.16 & 0.53 & 0.64 & 0.0095 \\
\hline CD133 Normal & 0.38 & 0.13 & -0.07 & 0.79 & -0.07 & 0.79 \\
\hline SOX2 Normal & 0.16 & 0.53 & 0.47 & 0.06 & 0.24 & 0.33 \\
\hline
\end{tabular}

Significantly concordant values are highlighted in bold.

ROI, region of interest.

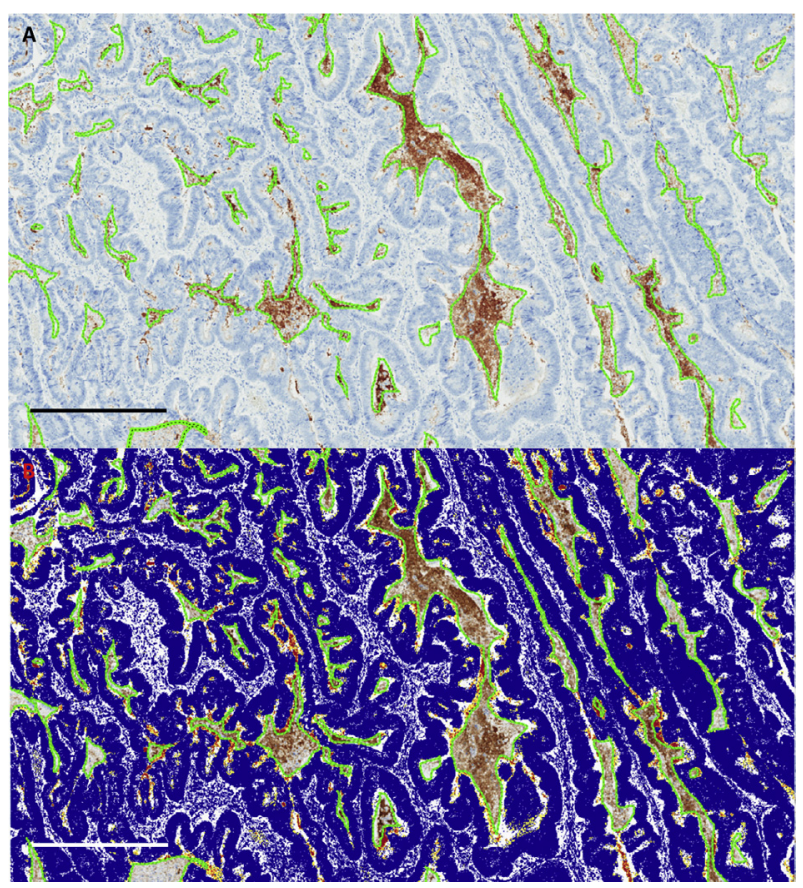

Fig. 3 Annotation and mark-up of tumour tissue for CD133 analysis. Lumina areas containing cell debris were excluded from analysis in the ROI method. Annotation was performed to exclude luminal cell debris without excluding true positive staining at the apical surface of positively staining cells. (A) Representative image for CD133 with manual annotations to exclude luminal areas. (B) Corresponding digital mark-up image of the same region. Red is strong positive staining, orange moderate, yellow weak and blue negative. $\mathrm{Bar}=500 \mu \mathrm{m}$

have been associated with disease progression or prognosis. The immunostains were analysed using tissue scoring and three automated digital analysis methods that analysed different tissue regions.

Overall, the digital analysis methods produced concordant results for the majority of immunostains performed in this study. However, CD133 staining in both tissue types and SOX2 staining in normal tissue revealed discordant results between the three methods. Pairwise comparisons revealed that only CD133 expression in tumour showed significantly concordant results. This finding was consistent with both the full-face and whole section methods showing a similar median percentage expression level (Table 4). The AC133 epitope has been reported previously to be expressed at the apical surface of tumour epithelial cells ${ }^{6,7,9}$ (Fig. 2F), with some studies scoring positively stained luminal areas as positive. The digital ROI method used here excluded these luminal areas, possibly explaining why lower expression levels were obtained with this method (Fig. 3).

CD133 and SOX2 expression in normal tissue showed discordant results in all pairwise comparisons, with the median expression levels from whole section analysis being considerably higher with both stains. This is likely due to the inclusion of background staining from muscle, nerve and necrotic tissues when analysing the entire section (Fig. 4). Results obtained with the manual ROI scoring method were considerably different from results obtained with the digital analysis methods when the rank of measured values for each method was compared (Table 1). A score of 0-3 for each region may have limited power to detect more subtle differences in marker expression levels when compared to the percentage expression or number of positive cells that can be 

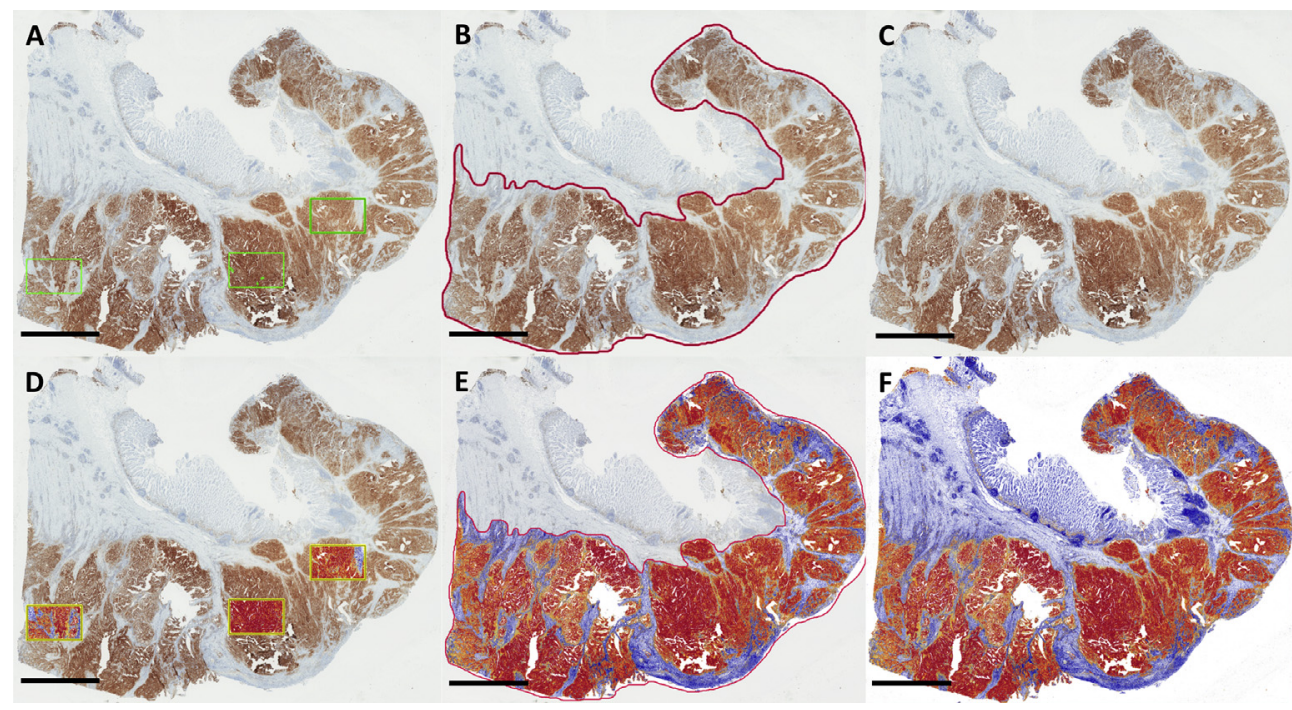

Fig. 4 Annotation and mark-up for the three digital analysis methods. Representative section stained with CD44v6 demonstrating the difference in analysis area for each digital method. Images demonstrate area selection for the ROI method (A,D), full-face method (B,E) and whole section method (C,F). (A-C) Annotated original IHC images for each method; (D-F) the mark-up image after digital analysis. Red is strong positive staining, orange moderate, yellow weak and blue negative. Bar $=5$ mm.

acquired from more objective digital analysis platforms. Scoring has also been associated with inter-observer variability, particularly for the interpretation of weak or intermediate expression intensity (i.e., +1 or +2 scores). ${ }^{3,4}$

When all four analysis methods were compared using $\mathrm{H}$ scores, a majority of the results for each stain were similar to that seen when percentage expression was compared for the three digital analysis methods (Table 5). In addition, CD133 in tumour tissue and SOX2 in normal tissue appeared to show an improvement in concordance (Table 5). However, when pairwise comparisons were performed the manual ROI scoring method and digital ROI scoring method demonstrated a high level of concordance for CD133 in tumour tissue, possibly explaining the improvement seen in the overall analysis (Supplementary Table 3, Appendix A). This result is not surprising considering the two methods are analysing expression in the same tissue regions.

The whole section and manual ROI scoring methods were the most rapid to complete, followed by the full-face and digital ROI methods. The grid-plot analysis method required approximately 30 minutes per region and hence was deemed not to be feasible. Studies that involve large numbers of patients often use TMA technology since it may not be feasible to perform full-face analysis of many samples. However, a greater rate of false-negative measurement has been reported for CSC marker expression evaluated using TMAs. ${ }^{10}$ Fullface analysis addresses this issue as a larger tissue area is analysed, and some tissue annotation to include areas of interest can also be completed within a reasonable time-frame.

Table 4 Median expression for samples that were discordant in Kendall's $\mathrm{W}$ overall test for the three digital analysis methods

\begin{tabular}{lccc}
\hline Method & CD133 Tumour & CD133 Normal & SOX2 Normal \\
\hline ROI & 2.35 & 1.79 & 2.13 \\
Full-face & 3.17 & 1.11 & 1.47 \\
Whole section & 3.26 & 6.18 & 5.8 \\
\hline
\end{tabular}

ROI, region of interest.
Although the digital methods used here can be considered objective, the expression levels of ALDH1, CD44v6, CD133 and Lgr5 were measured as a percentage of positive area whereas SOX2 was quantified as the percentage of positive cells. This could be considered a more appropriate method of measurement; however, the software used here was limited to the accurate counting of cells in which the marker was expressed in the nucleus. It is also important to note that expression for some of the markers was observed in cells of the stromal compartments in tumour samples. The software package used to analyse data in this study is unable to separately analyse tumour epithelium and stromal marker expression separately. However, recent biomarker studies have reported that CSC-associated gene expression in the stromal compartment of CRC tumours can provide valuable prognostic information. ${ }^{12,13}$ Therefore, these regions were not excluded from analysis in the current study. In addition, newer software packages are now coming to market that have the capability to analyse tumour epithelial and stromal areas separately which may provide more valuable prognostic information for future IHC marker studies. ${ }^{14}$

In conclusion, this study has objectively compared different methods for quantifying biomarker expression in tissue sections, both in terms of their feasibility and the quality of data obtained. For IHC studies, the selection of ROIs and the analysis method used can impact on the data

Table 5 Overall Kendall's W concordance coefficient test results for Hscores comparing the four analysis methods

\begin{tabular}{|c|c|c|c|c|}
\hline \multirow[t]{2}{*}{ Marker } & \multicolumn{2}{|c|}{ Tumour tissue } & \multicolumn{2}{|c|}{ Normal tissue } \\
\hline & Kendall's W & $p$ value & Kendall's W & $p$ value \\
\hline ALDH1 & 0.87 & $<0.001$ & 0.82 & $<0.001$ \\
\hline CD44V6 & 0.97 & $<0.001$ & 0.91 & $<0.001$ \\
\hline CD133 & 0.61 & 0.009 & 0.19 & 0.660 \\
\hline Lgr5 & 0.62 & 0.008 & 0.77 & 0.001 \\
\hline Sox 2 & 0.85 & $<0.001$ & 0.54 & 0.022 \\
\hline
\end{tabular}

Non-significant $p$ values indicate discordant results (bold). 
obtained and therefore the conclusions drawn. Thus, both factors should be considered during study design.

Acknowledgements: The authors would like to thank the staff of St John of God Pathology for sectioning and H\&E staining as well as Dr Tim Threlfall of the Western Australian Cancer Registry and Cheryl Penter of St John of God Subiaco Hospital for assistance with the data collection. We would also like to acknowledge the support of Nik Zeps from St John of God Hospital, Subiaco, throughout the study.

Conflicts of interest and sources of funding: This project was funded by the St John of God Foundation and the McCusker Charitable Foundation. TJM is funded by a University Postgraduate Award, UWA safety-net top-up and John Clauscen Murray Postgraduate Scholarship. The authors declare no conflicts of interest.

\section{APPENDIX A. SUPPLEMENTARY DATA}

Supplementary data related to this article can be found at http://dx.doi.org/10.1016/j.pathol.2016.09.063.

Address for correspondence: Tim Miller, Mail Bag 507, School of Surgery, University of Western Australia, 35 Stirling Highway, Crawley, WA 6009 , Australia. E-mail: timothy.miller@research.uwa.edu.au

\section{References}

1. O’Brien CA, Pollett A, Gallinger S, Dick JE. A human colon cancer cell capable of initiating tumour growth in immunodeficient mice. Nature 2007; 445: 106-10.
2. Ricci-Vitiani L, Lombardi DG, Pilozzi E, et al. Identification and expansion of human colon-cancer-initiating cells. Nature 2007; 445: 111-5.

3. Thomson TA, Hayes MM, Spinelli JJ, et al. HER-2/neu in breast cancer: Interobserver variability and performance of immunohistochemistry with 4 antibodies compared with fluorescent in situ hybridization. Mod Pathol 2001; 14: 1079-86.

4. Klarskov L, Ladelund S, Holck S, et al. Interobserver variability in the evaluation of mismatch repair protein immunostaining. Hum Pathol 2010; 41: 1387-96.

5. Elliott K, McQuaid S, Salto-Tellez M, Maxwell P. Immunohistochemistry should undergo robust validation equivalent to that of molecular diagnostics. J Clin Pathol 2015; 68: 766-70.

6. Horst D, Kriegl L, Engel J, Kirchner T, Jung A. CD133 expression is an independent prognostic marker for low survival in colorectal cancer. $\mathrm{Br}$ J Cancer 2008; 99: 1285-9.

7. Mia-Jan K, Jung SY, Kim I-Y, et al. CD133 expression is not an independent prognostic factor in stage II and III colorectal cancer but may predict the better outcome in patients with adjuvant therapy. BMC Cancer 2013; 13: 166.

8. Vaz MA, Martinez JC, Devesa JM, et al. Prognostic value of stem cell quantification in stage II colon cancer. PLoS One 2014; 9: e88480.

9. Kojima M, Ishii G, Atsumi N, Fujii S, Saito N, Ochiai A. Immunohistochemical detection of CD133 expression in colorectal cancer: A clinicopathological study. Cancer Sci 2008; 99: 1578-83.

10. Chen S, Song X, Chen Z, et al. CD133 expression and the prognosis of colorectal cancer: a systematic review and meta-analysis. PLoS One 2013; 8: 1-9.

11. Bland JM, Altman DG. Statistical methods for assessing agreement between two methods of clinical measurement. Lancet 1986; 1(8476) $307-10$.

12. Calon A, Lonardo E, Berenguer-Llergo A, et al. Stromal gene expression defines poor-prognosis subtypes in colorectal cancer. Nat Genet 2015; 47: 320-9.

13. Isella C, Terrasi A, Bellomo SE et al. Stromal contribution to the colorectal cancer transcriptome. Nat Genet 2015; 47: 312-9.

14. Schlederer M, Mueller KM, Haybaeck J, et al. Reliable quantification of protein expression and cellular localization in histological sections. PLoS One 2014; 9: e100822. 